Pacific Journal of Mathematics

DERIVATIONS OF OPERATOR ALGEBRAS INTO SPACES OF

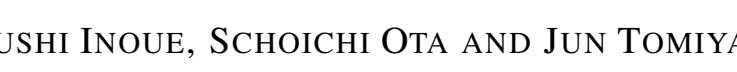




\title{
DERIVATIONS OF OPERATOR ALGEBRAS INTO SPACES OF UNBOUNDED OPERATORS
}

\author{
Atsushi Inoue, SchôIchi ÔTa AND Jun TomiYama
}

\begin{abstract}
This paper is to study the spatiality of unbounded derivations in operator algebras. Let $\mathscr{H}$ be a von Neumann algebra $\left(C^{*}\right.$-algebra) on a Hilbert space $(3)$ and $\delta$ be an unbounded derivation in $\mathscr{M}$. In this paper, extending $\delta$ to a derivation $\hat{\delta}$ of $\mathscr{M}$ into a certain space of unbounded operators, we study the spatiality of $\delta$ by investigating the property of $\hat{\delta}$.
\end{abstract}

1. Introduction. Unbounded derivations in operator algebras $\left(C^{*}\right.$-algebras and von Neumann algebras) have recently been investigated by many authors, since they are appeared as infinitesimal generators of strongly continuous one-parameter groups of *-automorphisms on $C^{*}$-algebras [see; 12]. In particular, the infinitesimal generator mentioned above is implemented by a symmetric operator by giving some representation of its $C^{*}$-algebra on a Hilbert space, and there exist many closed derivations in $C^{*}$-algebras which possess such a property [2]. In this point of view, we shall study the spatiality of unbounded derivations in operator algebras (see [2]; Problem). Our method is, roughly speaking, to examine the spatiality of an unbounded derivation $\delta$ in an operator algebra $\mathscr{C}$ by extending $\delta$ to a derivation of $\mathscr{l}$ into some space of unbounded operators containing $\mathscr{L}$.

Let $\mathscr{H}$ be a von Neumann algebra acting on a Hilbert space (5) and let $\delta$ be a ${ }^{*}$-derivation in $\mathscr{C}$ with $\sigma$-strongly dense domain $\mathscr{D}(\delta)$. Let $\mathscr{D}$ be a dense subspace of $\mathbb{S}$. We introduce various locally convex topologies in the space $\mathscr{L}^{\sharp}(\mathscr{D}$, (B) $)$ which is the set of all linear operators $T$ of $\mathscr{D}$ into $\mathscr{S}$ with $\mathscr{D}\left(T^{*}\right) \supset \mathscr{D}$, and extend $\delta$ to a $*$-derivation $\hat{\delta}$ of $\mathscr{A l}$ into $\mathscr{L}^{\sharp}(\mathscr{D},(\$)$ assuming corresponding continuity of $\delta$ in these topologies.

We shall then examine under what conditions the continuous *-derivation $\hat{\delta}$ of $\mathscr{C}$ into $\mathscr{L}^{\sharp}(\mathscr{D}$, (S) with some specified topology is spatial, i.e., there exists an element $H$ of $\mathscr{L}^{\sharp}(\mathscr{D}$, (B) $)$ such that $\hat{\delta}(A) \xi=[H, A] \xi=\{H A-A H\} \xi$ for all $A \in \mathscr{C l}$ and $\xi \in \mathscr{D}$. We call the dense subspace $\mathscr{D}$ countably dominated by a sequence $\left\{T_{n}\right\}$ of closed operators if $\mathscr{D}=\bigcap_{n=1}^{\infty} \mathscr{D}\left(T_{n}\right)$ and $\left\|T_{n} \xi\right\| \leqq\left\|T_{n+1} \xi\right\|$ for each $\xi \in \mathscr{D}$ and $n=1,2, \cdots$.

Our first result (Theorem 4.11) shows that if $\mathscr{C}$ is a left von Neumann algebra of a Hilbert algebra $\mathfrak{A}$ with identity and $\mathscr{D}$ is countably dominated by $\left\{T_{n}\right\}$ of closed operators then $\hat{\delta}$ is spatial. 
The second purpose of this paper is to show (Theorem 4.15) that if $\mathscr{C}$ has certain property (Definition 4.2) and $\mathscr{D}$ is countably dominated by $\left\{T_{n}\right\}$ of closed operators $\eta \mathscr{C}^{\prime}$ then $\hat{\delta}$ is a spatial *derivation of $\mathscr{C l}$ into $\mathscr{L}^{\sharp}(\mathscr{D},(\mathfrak{S})$.

2. Spaces of unbounded operators. Let \& be a Hilbert space with inner product $(\mid)$ and let $\mathscr{D}$ be a dense subspace of $\mathscr{B}$. We denote by $\mathscr{L}(\mathscr{D}, \mathbb{S})$ (resp. $\mathscr{L}_{c}(\mathscr{D}, \mathbb{S})$ ) the space of all (resp. closable) linear operators of $\mathscr{D}$ into $\mathbb{S}$ and by $\mathscr{L}^{\sharp}(\mathscr{D}, \mathbb{S})$ the space of operators $A$ in $\mathscr{L}(\mathscr{D}, \mathbb{S})$ for which there exists the adjoints $A^{*}$ whose domains $\mathscr{D}\left(A^{*}\right)$ contain $\mathscr{D}$. For each $T \in \mathscr{L}(\mathscr{D}, \mathbb{S})$ we define

$$
\|A\|_{T}=\sup _{\xi \in \mathscr{D}} \frac{\|A \xi\|}{\|T \xi\|}, \quad A \in \mathscr{L}(\mathscr{D},(\mathbb{S}),
$$

where $(\lambda / 0)=\infty$ for $\lambda>0$ and $(0 / 0)=0$,

$$
\mathfrak{M}_{T}=\left\{A \in \mathscr{L}(\mathscr{D}, \mathbb{S}) ;\|A\|_{T}<\infty\right\}
$$

and

$$
\mathfrak{M}_{T}^{\ddagger}=\left\{A \in \mathscr{L}^{\sharp}(\mathscr{D}, \mathbb{S}) ;\|A\|_{T}<\infty\right\} .
$$

Then it is easily seen that $\mathfrak{M}_{T}$ is a Banach space equipped with the norm $\|\cdot\|_{T}$ and $\mathfrak{M}_{T}^{\#}$ is a subspace of $\mathfrak{M}_{T}$.

The following lemma is an immediate consequence of the definitions of the spaces of $\mathfrak{M}_{T}$ and $\mathfrak{M}_{T}^{\#}$.

LEMmA 2.1. Let $T$ be an element of $\mathscr{L}^{\sharp}\left(\mathscr{D}\right.$, (S) such that $\overline{T^{-1}} \epsilon$ $\mathscr{B}(\mathbb{B})$, where $\mathscr{B}(\mathbb{S})$ denotes the algebra of all bounded linear operators on (S). We set

$$
\mathscr{B}_{T}=\left\{\overline{A T^{-1}} ; A \in \mathfrak{M}_{T}\right\} \quad \text { and } \quad \mathscr{B}_{T}^{\#}=\left\{\overline{A T^{-1}} ; A \in \mathfrak{M}_{T}^{\sharp}\right\} .
$$

Then the map $\phi: A \rightarrow \overline{A T^{-1}}$ is an isometric isomorphism of the Banach space $\mathfrak{M}_{T}$ onto the Banach space $\mathscr{B}(\mathbb{B})$.

LEMMA 2.2. Let \&S be a Hilbert space with inner product (|). If there exists a sequence $\left\{T_{n}\right\}$ of closed operators on \& such that

(1) $\mathscr{D}=\bigcap_{n=1}^{\infty} \mathscr{D}\left(T_{n}\right)$ is dense in (B);

(2) $\left\|T_{n} \xi\right\| \leqq\left\|T_{n+1} \xi\right\|$ for all $\xi \in \mathscr{D}$ and $n=1,2, \cdots$, then $\mathscr{L}^{\sharp}(\mathscr{D}, \mathbb{S})=\bigcup_{n=0}^{\infty} \mathfrak{M}_{T_{n}}^{\sharp}$ where $T_{0}=I$.

Proof. For each $\xi \in \mathscr{D}$ we set

$$
\|\xi\|_{T_{n}}=\left\|T_{n} \xi\right\| \text { for } n=0,1,2 \cdots .
$$

We consider the locally convex topology $t_{\left\{T_{n}\right\}}$ on $\mathscr{D}$ generated by 
family of the seminorms $\|\cdot\|_{T_{n}}(n=0,1,2, \cdots)$. Suppose that $\left\{\xi_{k}\right\}$ is a Cauchy sequence in $\left(\mathscr{D}, t_{\left\langle T_{n}\right\}}\right)$. Then we have

$$
\begin{array}{r}
\lim _{k \rightarrow \infty}\left\|\xi_{k}-\xi_{l}\right\|=0 \text { and } \lim _{k, l \rightarrow \infty}\left\|T_{n} \xi_{k}-T_{n} \xi_{l}\right\|=0 \\
\text { for } n=1,2, \cdots .
\end{array}
$$

Since $T_{n}$ is a closed operator, it follows that $x \in \mathscr{D}\left(T_{n}\right)$ and $\lim _{k \rightarrow \infty} T_{n} \xi_{k}=$ $T_{n} x$ for $n=1,2, \cdots$. Hence we have $x \in \bigcap_{n=1}^{\infty} \mathscr{D}\left(T_{n}\right)=\mathscr{D}$ and $\lim _{k \rightarrow \infty} T_{n} \xi_{k}=T_{n} x$ for $n=1,2, \cdots$. This implies that $\left(\mathscr{D}, t_{\left\{T_{n}\right\}}\right)$ is a Fréchet space.

Suppose $S \in \mathscr{L}^{\sharp}(\mathscr{D},(\mathbb{S})$. We show that the graph of $S: G(S) \equiv$ $\{\langle\xi, S \xi\rangle ; \xi \in \mathscr{D}\}$ is closed in $\left(\mathscr{D}, t_{\left\{T_{n}\right\}}\right) \times \mathbb{B}$. Suppose that a sequence $\left\{\left\langle\xi_{n}, S \xi_{n}\right\rangle\right\}$ in $G(S)$ converges to an element $\langle\xi, y\rangle$ of $\mathscr{D} \times \mathbb{B}$. It then follows that $\xi_{n}-\xi \in \mathscr{D}, \lim _{n \rightarrow \infty}\left\|\xi_{n}-\xi\right\|=0$ and $\lim _{n \rightarrow \infty} \| S\left(\xi_{n}-\xi\right)-$ $(y-S \xi) \|=0$. Since $S$ is closable, we have $y=S \xi$. This implies that $G(S)$ is closed in $\left(\mathscr{D}, t_{\left\{T_{n}\right\}}\right) \times \mathbb{B}$. By the closed graph theorem it follows that the map $S:\left(\mathscr{D}, t_{\left\{T_{n}\right\}}\right) \rightarrow \mathscr{B}$ is continuous. Hence there exist a number $n$ and a constant $\gamma>0$ such that

$$
\|S \xi\| \leqq \gamma\left\|T_{n} \xi\right\| \text { for all } \xi \in \mathscr{D} .
$$

Therefore, $S \in \mathfrak{M}_{T_{n}}^{\sharp}$. This implies that $\mathscr{L}^{\sharp}(\mathscr{D}, \mathbb{S})=\bigcup_{n=0}^{\infty} \mathfrak{M}_{T_{n}}^{\sharp}$.

Definition 2.3. Let $\mathscr{D}$ be a dense subspace in a Hilbert space (B). If there exists a sequence $\left\{T_{n}\right\}$ of closed operators in $\$ S$ such that $\mathscr{D}=\bigcap_{n=1}^{\infty} \mathscr{D}\left(T_{n}\right)$ and $\left\|T_{n} \xi\right\| \leqq\left\|T_{n+1} \xi\right\|$ for all $\xi \in \mathscr{D}$ and $n=1,2, \cdots$, then $\mathscr{D}$ is said to be countably dominated by $\left\{T_{n}\right\}$. If there exists a sequence $\left\{S_{n}\right\}$ in $\mathscr{L}^{\sharp}(\mathscr{D}, \mathbb{S})$ such that $\mathscr{L}^{*}(\mathscr{D}, \mathbb{S})=$ $\bigcup_{n=1}^{\infty} \mathfrak{M}_{S_{n}}^{*}$ and $\left\|S_{n} \xi\right\| \leqq\left\|S_{n+1} \xi\right\|$ for all $\xi \in \mathscr{D}$ and $n=1,2, \cdots$, then $\mathscr{L}^{\sharp}(\mathscr{D}, \mathbb{S})$ is said to be countably dominated by $\left\{S_{n}\right\}$.

REMARK. (1) Lemma 2.2 implies that if a pre-Hilbert space $\mathscr{D}$ is countably dominated then $\mathscr{L}^{\sharp}(\mathscr{D}, \mathbb{S})$ is also countably dominated.

(2) It will be seen, by a simple calculation, that if $\mathscr{L}^{\sharp}(\mathscr{D}, \mathbb{S})=$ $\bigcup_{n=1}^{\infty} \mathfrak{M}_{S_{n}}^{*}$ for $S_{n} \in \mathscr{L}^{\sharp}(\mathscr{D}) \equiv \mathscr{L}^{\sharp}(\mathscr{D}, \mathscr{D})(n=1,2, \cdots)$, then $\mathscr{L}^{\sharp}(\mathscr{D}$, (S) is countably dominated.

Let $\mathscr{D}$ be a dense subspace of a Hilbert space $\$$ s. We now introduce some locally convex topologies on $\mathscr{L}^{\sharp}(\mathscr{D}, \mathbb{S})$. We put

$$
\begin{aligned}
& P_{\xi, x}(A)=|(A \xi \mid x)|, \\
& P_{\xi}(A)=\|A \xi\|,
\end{aligned}
$$

where $A \in \mathscr{L}(\mathscr{D}, \mathbb{S}), \xi \in \mathscr{D}$ and $x \in(\mathbb{S}$. The locally convex topology on $\mathscr{L}(\mathscr{D}, \mathbb{S})$ generated by the seminorms $\left\{P_{\xi, \eta}(\cdot) ; \xi, \eta \in \mathscr{D}\right\}$ (resp. 
$\left.\left\{P_{\xi, x}(\cdot) ; \xi \in \mathscr{D}, x \in \mathbb{S}\right\},\left\{P_{\xi}(\cdot) ; \xi \in \mathscr{D}\right\}\right)$ is said to be the weak topology (resp. quasi-weak topology, strong topology) and is simply denoted by $t_{w}^{\mathscr{Q}}\left(\right.$ resp. $\left.t_{q w}^{\mathscr{g}}, t_{s}^{\mathscr{Q}}\right)$.

Let $\mathbb{S}_{\infty}$ be the Hilbert direct sum of the Hilbert spaces $\mathbb{S}_{n} \equiv$ $\mathbb{S}(n=1,2, \cdots)$ and let

$$
\begin{aligned}
\mathscr{D}_{\infty}(\mathscr{D})= & \left\{\left\{\xi_{n}\right\} \in \mathscr{B}_{\infty} ; \xi_{n} \in \mathscr{D} \text { for } n=1,2, \cdots\right. \\
& \text { and } \sum_{n=1}^{\infty}\left\|A \xi_{n}\right\|^{2}<\infty \text { for all } A \in \mathscr{L}^{\sharp}(\mathscr{D},(\mathbb{S})\} .
\end{aligned}
$$

We set

$$
\begin{aligned}
& P_{\left\{\xi_{n}\right\},\left\{x_{n}\right\}}(A)=\left|\sum_{n=1}^{\infty}\left(A \xi_{n} \mid x_{n}\right)\right|, \\
& P_{\left\{\xi_{n}\right\}}(A)=\left[\sum_{n=1}^{\infty}\left\|A \xi_{n}\right\|^{2}\right]^{1 / 2},
\end{aligned}
$$

where $A \in \mathscr{L}^{\sharp}(\mathscr{D}, \mathbb{S}),\left\{\xi_{n}\right\} \in \mathscr{D}_{\infty}(\mathscr{D})$ and $\left\{x_{n}\right\} \in \mathscr{D}_{\infty}$. We equip $\mathscr{L}^{\sharp}(\mathscr{D},(\mathfrak{S})$ with the locally convex topology $t_{\sigma w}^{\mathscr{D}}\left(\right.$ resp. $\left.t_{q \sigma w}^{\mathscr{D}}, t_{\text {os }}^{\mathscr{O}}\right)$ induced by the seminorms $\left\{P_{\left\{\xi_{n}\right\},\left\{\eta_{n}\right\}}(\cdot) ;\left\{\xi_{n}\right\},\left\{\eta_{n}\right\} \in \mathscr{D}_{\infty}(\mathscr{D})\right\}$ (resp. $\left\{P_{\left\{\xi_{n}\right\},\left\{x_{n}\right\}}(\cdot) ;\left\{\xi_{n}\right\} \in\right.$ $\left.\left.\mathscr{D}_{\infty}(\mathscr{D}),\left\{x_{n}\right\} \in \mathbb{S}_{\infty}\right\},\left\{P_{\left\{\xi_{n}\right\}}(\cdot) ;\left\{\xi_{n}\right\} \in \mathscr{D}_{\infty}(\mathscr{D})\right\}\right)$. The topology $t_{\sigma w}^{\mathscr{O}}$ (resp. $\left.t_{q \sigma w}^{\mathscr{O}}, t_{\sigma s}^{\mathscr{O}}\right)$ is said to be the $\sigma$-weak topology (resp. quasi- $\sigma$-weak topology, $\sigma$-strong topology) on $\mathscr{L}^{\sharp}(\mathscr{D}, \mathbb{S})$.

We next define the uniform topology and the quasi-uniform topology. A subset $\mathfrak{M}$ of $\mathscr{D}$ is said to be $\mathscr{D}$-bounded if

$$
\sup _{\xi \in \mathscr{N}}\|A \xi\|<\infty \quad \text { for each } A \in \mathscr{L}^{\sharp}(\mathscr{D},(\mathbb{S}) \text {. }
$$

We then define

$$
\begin{aligned}
P_{\mathfrak{M}}(A) & =\sup _{\xi \eta \mathfrak{M}}|(A \xi \mid \eta)|, \\
P^{\mathfrak{M}}(A) & =\sup _{\xi \in \mathfrak{M}}\|A \xi\|,
\end{aligned}
$$

where $\mathfrak{M}$ is $\mathscr{D}$-bounded and $A \in \mathscr{L}^{\sharp}(\mathscr{D}, \mathbb{S})$. The locally convex topology generated by the seminorms $\left\{P_{\mathfrak{M}}(\cdot) ; \mathfrak{M}\right.$ is $\mathscr{D}$-bounded $\}$ (resp. $\left\{P^{\Re}(\cdot) ; \mathfrak{M}\right.$ is $\mathscr{D}$-bounded $\left.\}\right)$ is said to be the uniform topology (resp. quasi-uniform topology) on $\mathscr{L}^{\sharp}(\mathscr{D}, \mathscr{S})$ and is simply denoted by $t_{u}^{\mathscr{O}}$ (resp. $\left.t_{q u}^{\mathscr{T}}\right)$.

We next define the $\rho$-topology and $\lambda$-topology on $\mathscr{L}^{\sharp}(\mathscr{D},(S)$. For each $T \in \mathscr{L}^{\sharp}(\mathscr{D}, \mathbb{S})$ we put

$$
\rho_{T}(A)=\sup _{\xi \in \mathscr{D}} \frac{|(A \xi \mid \xi)|}{\|T \xi\|^{2}}, \quad A \in \mathscr{L}^{\sharp}(\mathscr{D}, \mathbb{S}),
$$

where $(\lambda / 0)=\infty$ for $\lambda>0$ and $0 / 0=0$, and

$$
\mathfrak{R}_{T}^{\sharp}=\left\{A \in \mathscr{L}^{\sharp}(\mathscr{D}, \mathbb{S}) ; \rho_{T}(A)<\infty\right\} \text {. }
$$


Then it is easily seen that $\mathfrak{R}_{T}^{*}$ is a normed space equipped with the norm $\rho_{T}(\cdot)$ and $\mathscr{L}^{\sharp}(\mathscr{D}, \mathbb{S})=\bigcup_{T \in \mathscr{L}_{(\mathscr{O}, \mathbb{E})}} \Re_{T}^{\sharp}$. The inductive limit topology on $\mathscr{L}^{\sharp}(\mathscr{D}, \mathbb{S})$ with respect to the normed spaces $\left\{\left(\mathfrak{N}_{T}^{\sharp}, \rho_{T}(\cdot)\right)\right.$; $\left.T \in \mathscr{L}^{\sharp}(\mathscr{D}, \mathbb{S})\right\} \quad$ (resp. $\left.\left\{\left(\mathfrak{M}_{T}^{\#},\|\cdot\|_{T}\right) ; T \in \mathscr{L}^{\sharp}(\mathscr{D}, \mathbb{S})\right\}\right)$ is said to be the $\rho$-topology (resp. $\lambda$-topology) on $\mathscr{L}^{\sharp}(\mathscr{D}, \mathbb{S})$ and is denoted by $t_{\mathscr{O}}^{\mathscr{O}}$ (resp. $\left.t_{\lambda}^{\mathscr{T}}\right)$.

Now one may easily see the following lemma by the definitions of the topologies.

LEMMA 2.4. The relation among the topologies introduced here are as follows:

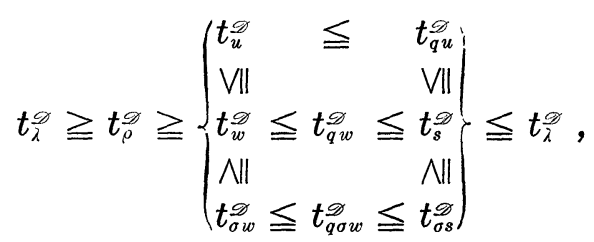

where the symbols $\tau_{1} \leqq \tau_{2}, \tau_{2} \geqq \tau_{1}$, N\|l and $\tau_{2}$ VIl mean the topology $\tau_{2}$ is finer than the topology $\tau_{1}$.

REMARK. The topologies $t_{u}^{\mathscr{\partial}}$ and $t_{q u}^{\mathscr{g}}$ (resp. the topologies $t_{\rho}^{\mathscr{\vartheta}}$ and $\left.t_{\lambda}^{\mathscr{Q}}\right)$ on $\mathscr{L}^{\sharp}(\mathscr{D},(\mathbb{S})$ are generalizations of the uniform topology and quasi-uniform one (resp. the $\rho$-topology and $\lambda$-topology) introduced by G. Lassner [8] (resp. D. Arnal and J. P. Jurzak [1]), for an unbounded operator algebra respectively. We denote by $t_{u}$ (resp. $t_{w}$, $t_{s}, t_{\sigma w}, t_{\sigma s}$ ) the usual uniform (resp. weak, strong, $\sigma$-weak, $\sigma$-strong) topology on $\mathscr{B}(\mathbb{S})$. The relations between the topologies on $\mathscr{B}(\mathbb{S})$

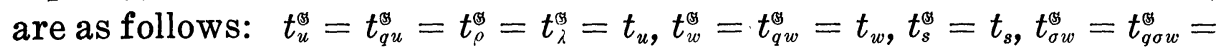
$t_{o w}$ and $t_{o s}^{\mathbb{s}}=t_{o s}$.

Lemma 2.5. Suppose that $\mathscr{L}^{\sharp}(\mathscr{D}, \mathbb{S})$ is countably dominated by $\left\{T_{n}\right\}$ and $\mathfrak{N}$ is a subset of $\mathscr{L}^{\sharp}(\mathscr{D}, \mathbb{S})$. Then the following statements are equivalent:

(1) $\mathfrak{R}$ is $t_{\rho}^{\mathscr{S}}$-bounded;

(2) $\mathfrak{R}$ is $t_{w}^{\mathscr{D}}$-bounded;

(3) there exist a number $n$ and a constant $\gamma>0$ such that

$$
|(A \xi \mid \xi)| \leqq \gamma\left\|\left(I+\left|\bar{T}_{n}\right|\right) \xi\right\| \text { for all } A \in \mathfrak{N} \text { and } \xi \in \mathscr{D},
$$

where $\bar{T}_{n}=U\left|\overline{T_{n}}\right|$ is the polar decomposition of $\overline{T_{n}}$.

Proof. This is proved in the same way as in ([13] Lemma 2.1). 
Lemma 2.6. Suppose that $\mathscr{L}^{\sharp}(\mathscr{D},(\mathbb{S})$ is countably dominated by $\left\{T_{n}\right\}$ and $\mathfrak{N}$ is a subset of $\mathscr{L}^{\sharp}(\mathscr{D}, \mathbb{S})$. Then the following statements are equivalent:

(1) $\mathfrak{N}$ is $t_{i}^{\mathscr{Q}}$-bounded;

(2) $\mathfrak{N}$ is $t_{q u}^{\mathscr{S}}$-bounded;

(3) $\mathfrak{N}$ is $t_{\sigma s}^{\mathscr{S}}$-bounded;

(4) there exists a number $n$ and a constant $\gamma>0$ such that $\|A \xi\| \leqq \gamma\left\|\left(I+\left|\bar{T}_{n}\right|\right) \xi\right\|$ for all $A \in \Re$ and $\xi \in \mathscr{D}$.

Furthermore, if $\mathscr{D}=\bigcap_{T \in \mathscr{L} \sharp(\mathscr{D} \leftrightarrow)} \mathscr{D}(\bar{T})$, then the statements $(1) \sim(4)$ are equivalent to the following statements (5) and (6):

(5) $\mathfrak{N}$ is $t_{s}^{\mathscr{O}}$-bounded;

(6) $\mathfrak{N}$ is $t_{q w}^{\curvearrowright}$-bounded.

Proof. Since $t_{\lambda}^{\mathscr{Q}} \geqq t_{q u}^{\mathscr{O}}$ and $t_{\lambda}^{\mathscr{O}} \geqq t_{\sigma s}^{\mathscr{O}}$, one can see the implications $(4) \Rightarrow(1),(1) \Rightarrow(2)$ and $(1) \Rightarrow(3)$. We show the implication $(3) \Rightarrow(4)$. Suppose that the statement (4) is not true. Then there exists a sequence $\left\{A_{n}\right\}$ in $\mathfrak{R}$ and a sequence $\left\{\xi_{n}\right\}$ of nonzero elements of $\mathscr{D}$ such that

$$
\left\|A_{n} \xi_{n}\right\| \geqq n^{2}\left\|\left(I+\left|\overline{T_{n}}\right|\right) \xi_{n}\right\| \quad \text { for } \quad n=1,2, \cdots .
$$

Putting

$$
\eta_{n}=\frac{\xi_{n}}{n\left\|\left(I+\left|\bar{T}_{n}\right|\right) \xi_{n}\right\|} \text { for } n=1,2, \cdots
$$

we have

$$
\left\|A_{n} \eta_{n}\right\| \geqq n \text { and }\left\|T_{n} \eta_{n}\right\|<\frac{1}{n}
$$

We now show $\left\{\eta_{n}\right\} \in \mathscr{D}_{\infty}(\mathscr{D})$. Since $\mathscr{L}^{\sharp}(\mathscr{D}, \mathscr{S})=\bigcup_{n=1}^{\infty} \mathfrak{M}_{T_{n}}^{\sharp}$, it follows that for each $A \in \mathscr{L}^{\sharp}(\mathscr{D}, \mathbb{S})$ there exists a number $k$ and a constant $\gamma>0$ such that

$$
\|A \xi\| \leqq \gamma\left\|T_{k} \xi\right\| \text { for all } \xi \in \mathscr{D} \text {. }
$$

Then we have

$$
\begin{aligned}
\sum_{n=1}^{\infty} \| & A \eta_{n}\left\|^{2} \leqq \gamma \sum_{n=1}^{\infty}\right\| T_{k} \eta_{n} \|^{2} \\
& \leqq \gamma\left\{\sum_{n=1}^{k-1}\left\|T_{k} \eta_{n}\right\|^{2}+\left\|T_{k} \eta_{k}\right\|^{2}+\left\|T_{k} \eta_{k+1}\right\|^{2}+\cdots\right\} \\
& \leqq \gamma\left\{\sum_{n=1}^{k-1}\left\|T_{k} \eta_{n}\right\|^{2}+\left\|T_{k} \eta_{k}\right\|^{2}+\left\|T_{k+1} \eta_{k+1}\right\|^{2}+\cdots\right\}
\end{aligned}
$$




$$
\begin{aligned}
& \leqq \gamma\left\{\sum_{n=1}^{k-1}\left\|T_{k} \eta_{n}\right\|^{2}+\frac{1}{k^{2}}+\frac{1}{(k+1)^{2}}+\cdots\right\} \\
& <\infty .
\end{aligned}
$$

This means $\left\{\eta_{n}\right\} \in \mathscr{D}_{\infty}(\mathscr{D})$. Furthermore, we have

$$
\begin{aligned}
\sup _{A \in \Re} P_{\left\{\eta_{n}\right\}}(A) & =\sup _{A \in \Re}\left[\sum_{n=1}^{\infty}\left\|A \eta_{n}\right\|^{2}\right]^{1 / 2} \\
& \geqq\left\|A_{n} \eta_{n}\right\| \geqq n .
\end{aligned}
$$

This contradicts that $\mathfrak{R}$ is $t_{\sigma s}^{\mathscr{g}}$-bounded. This comqletes the proof of the implication $(3) \Rightarrow(4)$.

The implication $(2) \Rightarrow(4)$ is proved in the same way as in ([13] Lemma 2.2).

If $\mathscr{D}=\bigcap_{T \in \mathscr{L} \sharp(\mathscr{O},(\mathbb{B}))} \mathscr{D}(\bar{T})$, the equivalence of the statements $(1) \sim$ (6) follows from ([1] Proposition 1.6).

3. Extension of derivations. Let $\mathscr{C}$ be a $C^{*}$-algebra (or a von Neumann algebra). A linear map $\delta: \mathscr{D}(\delta) \subset \mathscr{C} \rightarrow \mathscr{C}$ is said to be a $*$-derivation in $\mathscr{C l}$ if it satisfies the following conditions:

(1) the domain $\mathscr{D}(\delta)$ of $\delta$ is a dense *-subalgebra of $\mathscr{C}$ (i.e., $\mathscr{D}(\delta)$ is norm-dense if $\mathscr{C}$ is a $C^{*}$-algebra, and weak-dense if $\mathscr{C}$ is a von Neumann algebra);

(2) $\delta(A B)=\delta(A) B+A \delta(B)$ for each $A, B \in \mathscr{D}(\delta)$;

(3) $\delta\left(A^{*}\right)=\delta(A)^{*}$ for each $A \in \mathscr{D}(\delta)$.

We begin with the following lemma.

Lemma 3.1. Let $\mathscr{C l}$ be a unital $C^{*}$-algebra acting on a Hilbert space $\mathbb{B}$ and let $\delta$ be a *-derivation in $\mathscr{M}$ with domain $\mathscr{D}(\delta)$. If there exists a dense subspace $\mathscr{D}$ of $\$ S$ such that $\mathscr{M} \mathscr{D} \subset \mathscr{D}$ and $\delta$ is a continuous map of $\left(\mathscr{D}(\delta), t_{u}\right)$ into $\left(\mathscr{K}, t_{q u}^{\mathscr{P}}\right)$, then $\delta$ is extended to a continuous linear map $\hat{\delta}$ of $\left(\mathscr{L}, t_{u}\right)$ into $\left(\mathscr{L}^{\sharp}(\mathscr{D}, \mathbb{S}), t_{q u}^{\mathscr{g}}\right)$ such that

(1) $\hat{\delta}(A B) \xi=\hat{\delta}(A) B \xi+A \hat{\delta}(B) \xi$

(2) $\hat{\delta}(A)^{*} \xi=\hat{\delta}\left(A^{*}\right) \xi$;

(3) $\hat{\delta}\left(A^{*}\right)^{*} C \xi=C \hat{\delta}(A) \xi$

for each $A, B \in \mathscr{A}, C \in \mathscr{C}^{\prime}$ and $\xi \in \mathscr{D}$. Namely, the following diagram holds:

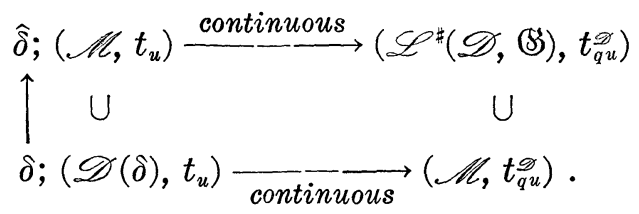

By Lemma 3.1 we define a derivation of a $C^{*}$-algebra into a space of unbounded operators as follows: 
Definition 3.2. Let $\mathscr{D}$ be a dense subspace in a Hilbert space (S) and let $\mathscr{C l}$ be a unital $C^{*}$-algebra acting on (S) with $\mathscr{H} \mathscr{D} \subset \mathscr{D}$. A linear map $\delta$ of $\mathscr{C}$ into $\mathscr{L}(\mathscr{D}, \mathbb{S})$ is said to be a derivation of $\mathscr{l l}$ into $\mathscr{L}(\mathscr{D},(B))$ if

$$
\delta(A B) \xi=\delta(A) B \xi+A \delta(B) \xi \text { for each } A, B \in \mathscr{M l} \text { and } \xi \in \mathscr{D} \text {. }
$$

In particular, a derivation $\delta$ is said to be a *-derivation if the range of $\delta$ is contained in $\mathscr{L}^{*}(\mathscr{D},(3)$ and

$$
\delta(A)^{*} \xi=\delta\left(A^{*}\right) \xi \text { for each } A \in \mathscr{C l} \text { and } \xi \in \mathscr{D} \text {. }
$$

If a derivation $\delta$ of $\mathscr{C l}$ into $\mathscr{L}(\mathscr{D},(S)$ is a continuous map of

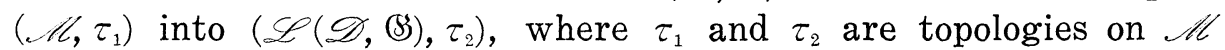
and $\mathscr{L}\left(\mathscr{D}\right.$, (S) respectively, then it is said to be $\left(\tau_{1} \rightarrow \tau_{2}\right)$-continuous.

We also have the following result:

Lemma 3.3. Let $/ l$ be a von Neumann algebra acting on a Hilbert space $\$ 3$ and let $\delta$ be a $*$-derivation in $/ l$. If $\delta$ is $\left(t_{w} \rightarrow t_{q w}^{\mathscr{O}}\right)$ continuous (resp. $\left(t_{s} \rightarrow t_{s}^{\mathscr{\sigma}}\right),\left(t_{\sigma w} \rightarrow t_{q \sigma w}^{\mathscr{g}}\right),\left(t_{\sigma s} \rightarrow t_{\sigma s}^{\mathscr{g}}\right)$-continuous), then $\delta$ is extended to $a\left(t_{w} \rightarrow t_{q w}^{\mathscr{g}}\right)$-continuous $\left(\mathrm{resp} .\left(t_{s} \rightarrow t_{q \sigma w}^{\mathscr{g}}\right),\left(t_{\sigma w} \rightarrow t_{q \sigma w}^{\mathscr{g}}\right),\left(t_{\sigma s} \rightarrow t_{\sigma s}^{\mathscr{S}}\right)-\right.$ continuous) *-derivation $\hat{\delta}$ of $\mathscr{L}$ into $\mathscr{L}^{*}\left(\mathscr{D}\right.$, (S) satisfying $\hat{\delta}\left(A^{*}\right)^{*} C \xi=$ $C \hat{\delta}(A) \xi$ for each $A \in \mathscr{l l}, C \in \mathscr{M}^{\prime}$ and $\xi \in \mathscr{D}$.

Definition 3.4. Let $\mathscr{D}$ be a dense subspace of a Hilbert space (S) and let $\delta$ be a $*$-derivation of a $C^{*}$-algebra $\mathscr{C}$ on $(5)$ into $\mathscr{L}^{\sharp}(\mathscr{D}$, (S)). If $\delta(\mathscr{C}) \subset \mathfrak{M}_{T}^{*}$ for some $T \in \mathscr{L}^{\sharp}(\mathscr{D}$, (B)), then $\delta$ is said to be a *derivation of $\mathscr{C}$ into $\mathfrak{M}_{T}^{ \pm}$. If there exists an element $T$ of $\mathscr{L}^{\sharp}(\mathscr{D}, \mathbb{B})$ such that $\delta\left(\mathscr{C}_{u}\right)$ is a bounded subspace of the normed space $\mathfrak{M}_{T}^{\#}$, where $\mathscr{C}_{u}$ is the set of all unitary operators in $\mathscr{A}$, then $\delta$ is said to be quasi-bounded.

Lemma 3.5. Let $\mathscr{C l}_{\text {be }}$ a unital $C^{*}$-algebra acting on a Hilbert space (S) and let $\delta$ be a *-derivation in $\mathscr{L}$. If there exist a dense subspace $\mathscr{D}$ of $(S)$ and an element $T$ of $\mathscr{L}^{*}(\mathscr{D}$, (S) such that $\mathscr{L} \mathscr{D} \subset$ $\mathscr{D}$ and $\|\delta(A)\|_{T} \leqq\|A\|$ for all $A \in \mathscr{D}(\delta)$, then $\delta$ is extended to a quasi-bounded *-derivation $\hat{\delta}$ of $\mathscr{C}$ into $\mathfrak{M}_{\bar{T}}^{*}$ satisfying $\hat{\delta}\left(A^{*}\right)^{*} C \xi=$ $C \hat{\delta}(A) \xi$ for each $A \in, \mathbb{l}, C \in, \mathbb{l}^{\prime}$ and $\xi \in \mathscr{D}$.

We now give some examples of quasi-bounded *-derivations.

ExAMPLe 3.6. Let $\delta$ be a spatial derivation in a $C^{*}$-algebra $\mathscr{C}$ acting on a Hilbert space $\mathbb{B}$ with domain $\mathscr{D}(\delta)$, i.e., there exists a symmetric operator $H$ on $(S)$ such that $\mathscr{D}(\delta) \mathscr{D}(H) \subset \mathscr{D}(H)$ and $\delta(A) \xi=$ $i[H, A] \xi$ for each $A \in \mathscr{D}(\delta)$ and $\xi \in \mathscr{D}(H)$. If there exists a closed 
operator $T \eta \mathscr{C l}^{\prime}$ and a constant $\gamma>0$ such that $\|H \xi\| \leqq \gamma\|T \xi\|$ for all $\xi \in \mathscr{D}(T)$, then $\delta$ is extended to a quasi-bounded $*$-derivation $\hat{\delta}$ of $\mathscr{L}$ into $\mathscr{L}^{\sharp}(\mathscr{D}(T),(S)$.

2. Let $\mathscr{A}_{i}$ be a von Neumann algebra on a Hilbert space $\mathbb{S}_{i}$ and let $\delta_{i}$ be a bounded *-derivation on $\mathscr{M}_{i}(i=1,2, \cdots)$. Let $\mathscr{C}$ be a direct sum of the von Neumann algebras $\mathscr{M}_{i}$ and let $\$ S$ be the direct sum of the Hilbert spaces $\mathbb{S}_{i}$. We define

$$
\begin{gathered}
\mathscr{D}(\delta)=\left\{A=\left(A_{i}\right) \in \prod_{i} \mathscr{C}_{i} ; A_{i} \neq 0 \text { for only finite coordinates }\right\}, \\
\delta(A)=\left(\delta_{i}\left(A_{i}\right)\right), \quad A=\left(A_{i}\right) \in \mathscr{D}(\delta) .
\end{gathered}
$$

Then $\delta$ is a $*$-derivation in $\mathscr{C}$ with the weakly dense domain $\mathscr{D}(\delta)$, but it is not generally bounded. However, $\delta$ is $\left(t_{w} \rightarrow t_{q w}^{\mathscr{S}}\right)$-continuous (and $\left(t_{s} \rightarrow t_{s}^{\mathscr{O}}\right),\left(t_{u} \rightarrow t_{u}^{\mathscr{I}}\right),\left(t_{u} \rightarrow t_{q u}^{\mathscr{T}}\right),\left(t_{u} \rightarrow t_{\lambda}^{\mathscr{V}}\right),\left(t_{u} \rightarrow t_{\tilde{\nu}}^{\mathscr{O}}\right)$-continuous), where

$$
\mathscr{D}=\left\{\left(\xi_{i}\right) \in \mathscr{B} ; \xi_{i} \neq 0 \text { for only finite coordinates }\right\} \text {. }
$$

Putting

$$
T=\left(\left\|\delta_{i}\right\| I_{i}\right)
$$

where $\left\|\delta_{i}\right\|$ is the norm of $\delta_{i}$ and $I_{i}$ is the identity operator on $\mathbb{S}_{i}$, we have

$$
\|\delta(A) \xi\| \leqq\|A\|\|T \xi\| \text { for each } A \in \mathscr{D}(\delta) \text { and } \xi \in \mathscr{D} .
$$

Hence, $\delta$ is extended to a quasi-bounded *-derivation of $\mathscr{C l}$ into $\mathfrak{M}_{T}^{\sharp}$.

3. Let $\delta$ be a $\left(t_{u} \rightarrow t_{w}^{\mathscr{Q}}\right)$-continuous $*$-derivation of $\mathscr{M}$ into $\mathscr{L}^{\sharp}(\mathscr{D})\left(\equiv \mathscr{L}^{\sharp}(\mathscr{D}, \mathscr{D})\right)$. If $\delta(\mathscr{L})$ is a finite dimensional subspace of $\mathscr{L}^{\sharp}(\mathscr{D})$, then $\delta$ is a quasi-bounded $*$-derivation of $\mathscr{C l}$ into $\mathscr{L}^{\sharp}(\mathscr{D}$, (S).

4. Let $\delta$ be a *-derivation in a $C^{*}$-algebra $\mathscr{C}$ acting on a Hilbert space $(\$)$. If there exists a densely defined closed operator $T$ on (S) such that $\mathscr{C P}(T) \subset \mathscr{D}(T)$ and $\delta$ is $\left(t_{u} \rightarrow t_{q u}^{\mathscr{g}(T)}\right)$-continuous (or $\left(t_{u} \rightarrow t_{i}^{\vartheta(T)}\right)$-continuous), then $\delta$ is extended to a quasi-bounded *-derivation of $\mathscr{l}$ into $\mathscr{L}^{\sharp}(\mathscr{D}(T)$, (S). This follows immediately from Lemma 2.2 .

As a slight generalization of Example 3.6, 4 we have the following result:

Lemma 3.7. Let $\mathscr{D}$ be a countably dominated subspace in a Hilbert space \&s by a sequence $\left\{T_{n}\right\}$ of closed operators on \&. If $\delta$ is a $\left(t_{u} \rightarrow t_{q u}^{\mathscr{O}}\right)$-continuous (or $\left(t_{u} \rightarrow t_{\bar{\lambda}}^{\mathscr{O}}\right),\left(t_{w} \rightarrow t_{q w}^{\mathscr{O}}\right),\left(t_{\sigma w} \rightarrow t_{q \sigma w}^{\mathscr{O}}\right),\left(t_{s} \rightarrow t_{s}^{\mathscr{O}}\right)$, $\left(t_{\text {os }} \rightarrow t_{\text {os }}^{:}\right)$-continuous) $*$-derivation of $\mathscr{M}$ into $\mathscr{L}^{\#}(\mathscr{D}$, (S), then $\delta$ is quasi-bounded. 
Proof. Suppose that $\delta$ is $\left(t_{u} \rightarrow t_{q u}^{\mathscr{O}}\right)$-continuous. By the continuity of $\delta, \delta\left(\mathscr{M}_{1}\right)$ is a bounded subset of $\left(\mathscr{L}^{\sharp}(\mathscr{D}, \mathscr{S}), t_{q u}^{\mathscr{g}}\right)$, where $\mathscr{M}_{1}$ is the unit ball of $\mathscr{M}$. It then follows from Lemma 2.4 that $\delta\left(\mathscr{M}_{1}\right)$ is a bounded subset of the normed space $\mathfrak{M}_{I+\left|T_{n}\right|}^{\sharp}$ for some $n$. This implies that $\delta$ is quasi-bounded.

4. The spatiality of quasi-bounded $*$-derivations. Throughout this section we may assume that $\mathscr{D}$ is a dense subspace of a Hilbert space $(B)$ and $\mathscr{C}$ is a unital $C^{*}$-algebra with $\mathscr{M D} \subset \mathscr{D}$. Let $\delta$ be a quasi-bounded *-derivation of $\mathscr{C}$ into $\mathscr{L}^{\sharp}(\mathscr{D}$, (S), i.e., there exists an element $T$ of $\mathscr{L}^{\sharp}(\mathscr{D}, \mathbb{S})$ such that $\overline{T^{-1}} \in \mathscr{B}(\mathbb{S})$ and $\delta\left(\mathscr{C}_{u}\right)$ is a bounded subset of the normed space $\mathfrak{M}_{T}^{\#}$.

Lemma 4.1. Suppose that $\mathfrak{M}$ is a subspace of $\mathscr{L}(\mathscr{D}, \mathbb{S})$. Then the following statements are equivalent:

(1) $f$ is a $t_{q w}^{\mathscr{S}}$-continuous linear functional on $\mathfrak{M}$;

(2) $f$ is a $t_{s}^{\mathscr{g}}$-continuous linear functional on $\mathfrak{M}$;

(3) $f=\sum_{i=1}^{n} \omega_{\xi_{i}, x_{i}}$ for $\xi_{i} \in \mathscr{D}$ and $x_{i} \in \mathbb{S}$, where $\omega_{\xi, x}(A)=(A \xi \mid x)$ for $A \in \mathscr{L}(\mathscr{D}, \mathbb{S}), \xi \in \mathscr{D}$ and $x \in \mathbb{B}$.

Proof. This is proved in the same way as in ([1] Theorem 1.3).

Let $T \in \mathscr{L}^{\sharp}(\mathscr{D}, \mathbb{S})$ and $\overline{T^{-1}} \in \mathscr{B}(\mathbb{S})$. Then, by Lemma $2.1 \mathscr{B}_{T}^{\#} \equiv$ $\left\{\overline{A T^{-1}} ; A \in \mathfrak{M}_{T}^{\sharp}\right\}$ is a subspace of $\mathscr{B}(\mathbb{S})$. We denote by $\widetilde{\mathscr{B}}_{T}^{\sharp}$ the $t_{w^{-}}$ closure of $\mathscr{B}_{T}^{\#}$ and denote by $\widetilde{\mathfrak{M}}_{T}^{\sharp}$ the $t_{q w}^{\mathscr{g}}$-closure of $\mathfrak{M}_{T}^{\sharp}$ in $\mathscr{L}(\mathscr{D}$, (B)). Then $\tilde{\mathscr{B}}_{T}^{\#}$ is a weakly closed subspace of $\mathscr{B}(\mathbb{S})$ and $\tilde{\mathfrak{M}}_{T}^{\#}$ is $t_{q w}^{\mathscr{Q}}$-closed subspace of $\mathscr{L}(\mathscr{D},(\mathbb{S})$. Furthermore, the following lemma is seen by a simple calculation.

LEMMA 4.2. Let $\phi$ be the isomorphism of $\mathfrak{M}_{T}^{\#}$ onto $\mathscr{B}_{T}^{\sharp}$ in Lemma 2.1. Then $\phi^{-1}$ is a continuous map of $\left(\mathscr{B}_{T}^{\#}, t_{w}\right)$ onto $\left(\mathfrak{M}_{T}^{\#}, t_{q w}^{\mathscr{O}}\right)$, so that it is extended to a continuous linear map $\tilde{\phi}^{-1}$ of $\left(\tilde{\mathscr{B}}_{T}^{\#}, t_{w}\right)$ onto $\left(\widetilde{\mathfrak{M}}_{T}^{\sharp}, t_{q w}^{\mathscr{g}}\right)$.

Lemma 4.3. Let $\Omega$ be a subset of $\mathfrak{M}_{T}^{\sharp}$ and let $\mathfrak{Q}$ be the $t_{q w}^{\mathscr{O}}$-closed convex hull of $\Omega$ in $\mathscr{L}\left(\mathscr{D},(\mathbb{S})\right.$. If $\Omega$ and $\Re^{*} \equiv\left\{A^{\#}=A^{*} / \mathscr{D} ; A \in \Re\right\}$ are bounded in $\mathfrak{M}_{T}^{\#}$, where $A^{*} / \mathscr{D}$ is the restriction of $A^{*}$ to $\mathscr{D}$, then $\mathfrak{Q}$ is a $t_{q w}^{\mathscr{O}}$-compact subset of $\mathfrak{M}_{T}^{\sharp}$.

Proof. Let $\Omega^{\prime}$ be the convex hull of $\Re$. Then $\Re^{\prime}$ and $\left(\Omega^{\prime}\right)^{*}$ are bounded in $\mathfrak{M}_{T}^{\#}$. Hence we may assume that $\Omega$ is convex. We first show that $\mathfrak{Q}$ is a bounded subset of the normed space $\mathfrak{M}_{T}^{\#}$. By the boundedness of $\Omega$ and $\Omega$ there exists a constant $\gamma>0$ such that $\|A\|_{T} \leqq \gamma$ and $\left\|A^{*}\right\|_{T} \leqq \gamma$ for all $A \in \Re$. For each $S \in \mathfrak{Q}$ there is a 
net $\left\{A_{\alpha}\right\}$ in $\Omega$ which converges to $S$ with respect to the topology $t_{q w}^{\mathscr{g}}$. It then follows that for each $\xi \in \mathscr{D}$ and $x \in \mathbb{B S}$

$$
\begin{aligned}
|(S \xi \mid x)| & =\lim _{\alpha}\left|\left(A_{\alpha} \xi \mid x\right)\right| \\
& \leqq \varlimsup_{\alpha}\left\|A_{\alpha} \xi\right\|\|x\| \\
& \leqq \gamma\|T \xi\|\|x\|,
\end{aligned}
$$

so that $\|S\|_{T} \leqq \gamma$. Furthermore, for each $\xi, \eta \in \mathscr{D}$ we have

$$
\begin{aligned}
|(S \xi \mid \eta)| & =\lim _{\alpha}\left|\left(A_{\alpha} \xi \mid \eta\right)\right| \\
& \leqq \varlimsup_{\alpha}\left\|A_{\alpha}^{*} \eta\right\|\|\xi\| \\
& \leqq \gamma\|T \eta\|\|\xi\| .
\end{aligned}
$$

Hence, $\eta \in \mathscr{D}\left(S^{*}\right)$. Thus we have $S \in \mathfrak{M}_{T}^{\sharp}$ and $\|S\|_{T} \leqq \gamma$.

We show that $\mathfrak{Q}$ is a $t_{q w}^{\mathscr{P}}$-compact subset of $\mathfrak{M}_{T}^{\sharp}$. In fact, $\left(\widetilde{\mathscr{B}}_{T}^{\sharp}\right)_{r} \equiv$ $\left\{X \in \widetilde{\mathscr{B}}_{T}^{\sharp} ;\|X\| \leqq \gamma\right\}$ is weakly compact, and so Lemma 4.2 implies that

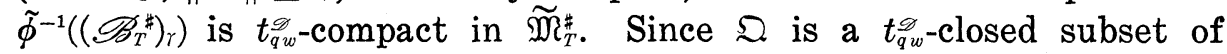
$\tilde{\phi}^{-1}\left(\left(\widetilde{\mathscr{B}}_{T}^{\sharp}\right)_{r}\right)$, it follows that $\mathfrak{Q}$ is a $t_{q w}^{\mathscr{O}}$-compact subset of $\mathfrak{M}_{T}^{\sharp}$.

Notation. Let $\Re_{\delta}$ be a set $\left\{U^{*} \delta(U) ; U \in \mathscr{M}_{u}\right\}$ and let $\mathfrak{O}_{\delta}$ be the $t_{q w}^{\mathscr{g}}$-closed convex hull of $\Re_{i}$ in $\mathscr{L}(\mathscr{D},(\mathbb{S})$.

LeMma 4.4. $\mathfrak{D}_{\delta}$ is a $t_{q \omega}^{\mathscr{O}}$-compact subset of $\mathfrak{M}_{T}^{\sharp}$.

Proof. It is easily seen that $\Re_{\tilde{o}}$ and $\Re_{\delta}^{*}$ are bounded subsets of $\mathfrak{M}_{T}^{\sharp}$. Hence, the lemma follows from Lemma 4.3.

Furthermore, one may easily see the following lemma.

LEMma 4.5. For each $U \in \mathscr{K}_{u}$ we define

$$
A_{U}(S)=U^{*} S U+U^{*} \delta(U) \quad \text { for } \quad S \in \mathscr{L}^{\sharp}(\mathscr{D},(\mathfrak{S}) .
$$

Then;

(1) $A_{U}$ is a $t_{q w}^{\mathscr{D}}$-continuous affine map of $\mathscr{L}^{\sharp}\left(\mathscr{D}\right.$, (S) into $\mathscr{L}^{\sharp}(\mathscr{D}$, (S));

(2) $A_{U}\left(V^{*} \delta(V)\right)=(V U)^{*} \delta(V U)$ for each $U, V \in \mathscr{C l}_{u}$;

(3) $A_{U} \mathfrak{Q}_{\hat{\jmath}} \subset \mathfrak{Q}_{\delta}$ for each $U \in \mathscr{A}_{u}$;

(4) $A_{U} A_{V}=A_{V U}$ for each $U, V \in \mathscr{M}_{u}$.

Hence, $G_{\delta, T} \equiv\left\{A_{u} ; U \in \mathscr{A}_{u}\right\}$ is a semigroup of $t_{q w}^{\mathscr{g}}$-continuous affine maps of $\mathfrak{\Omega}_{\hat{o}}$ into $\mathfrak{\Omega}_{\hat{\delta}}$.

Definition 4.6. If for each pair of elements $S_{1} \neq S_{2}$ in $\mathcal{B S}_{\delta}$ the $t_{s}^{\mathscr{Q}}$-closure of $\left\{A_{U}\left(S_{1}\right)-A_{U}\left(S_{2}\right) ; U \in \mathscr{A}_{u}\right\}$ does not contain 0 , then $G_{\tilde{\delta}, T}$ is said to be noncontracting. 
Definition 4.7. Let $\mathscr{D}$ be a dense subspace of a Hilbert space (B) and let $\mathscr{C}$ be a $C^{*}$-algebra acting on $B$ with $\mathscr{C D} \subset \mathscr{D}$. A *derivation (resp. a derivation) $\delta$ of $\mathscr{L}$ into $\mathscr{L}^{*}(\mathscr{D}$, (S) $($ resp. $\mathscr{L}(\mathscr{D}$, (S)) is said to be spatial if there exists an element $H$ of $\mathscr{L}^{*}(\mathscr{D}$, (S)(resp. $\mathscr{L}(\mathscr{D},(3))$ such that

$$
\delta(A) \xi=[H, A] \xi \text { for all } A \in \mathscr{C l} \text { and } \xi \in \mathscr{D} .
$$

Proposition 4.8. If $G_{\delta, T}$ is noncontracting, then there exists an element $S$ of $\mathfrak{D}_{\hat{\delta}}$ such that

$$
\delta(A) \xi=[S, A] \xi \text { for all } A \in \mathscr{L l} \text { and } \xi \in \mathscr{D} ;
$$

that is, $\delta$ is spatial.

Proof. We consider the locally convex space $\mathscr{X}^{7}=\left(\mathscr{L}^{*}\left(\mathscr{D},(\mathbb{S}), t_{s}^{\mathscr{S}}\right)\right.$. By Lemma 4.1 we have $\sigma\left(\mathscr{X}, \mathscr{X}^{*}\right)=t_{q w}^{\mathscr{O}}$, and hence it follows from Lemmas $4.4,4.5$ that $\mathfrak{D}_{\hat{o}}$ is a weakly compact subset of $\mathscr{Z}$ and $G_{\delta, T}$ is a noncontracting semigroup of weakly continuous affine maps of $\mathfrak{\Omega}_{\tilde{0}}$ into $\mathfrak{\Omega}_{\tilde{\delta}}$. By Ryll-Nardzewski's fixed point theorem [9] there exists an element $S_{0}$ of $\mathfrak{D}_{\tilde{o}}$ such that

$$
A_{U}\left(S_{0}\right)=S_{0} \text { for all } U \in \mathscr{C}_{l} .
$$

Hence, putting $S=-S_{0}$, we have

$$
\delta(A) \xi=[S, A] \xi \text { for all } A \in \mathscr{C l} \text { and } \xi \in \mathscr{D} .
$$

Corollary 4.9. Let $\mathscr{D}$ be a countably dominated subspace of a Hilbert space (S) and let $\mathscr{C}$ be a commutative $C^{*}$-algebra acting on (3) with $\mathscr{A D} \subset \mathscr{D}$. Then there does not exist any nonzero $\left(t_{w} \rightarrow t_{\bar{q} w}\right)$ -

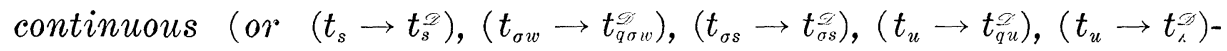
continuous) *-derivation in $\mathscr{C}$.

Proof. Suppose that $\delta$ is a $*$-derivation which is continuous in one of the above topologies. It then follows from Lemma 3.3 that $\delta$ is extended to a quasi-bounded $*$-derivation $\hat{\delta}$ of $\mathscr{C l}$ into $\mathfrak{M}_{T}^{\sharp}$ where $T \in \mathscr{L}^{\sharp}\left(\mathscr{D}\right.$, (S) and $\overline{T^{-1}} \in \mathscr{B}(\mathbb{S})$. Since $\mathscr{C}$ is commutative, we can easily see that the semigroup $G_{\hat{v} T}$ is noncontracting. Hence it follows from Proposition 4.8 that there exists an element $H$ of $\mathfrak{Q}_{\hat{o}}$ such that $\hat{\delta}(A) \xi=[H, A] \xi$ for all $A \in \mathscr{C}$ and $\xi \in \mathscr{D}$. By Lemma 3.3 the elements $A$ and $H$ commute, and so $\hat{\delta}=0$.

LEMMA 4.10. Let \&s be the completion of a maximal Hilbert algebra $\mathfrak{A}$ with identity $e$ and let $\mathscr{C}$ be the left von Neumann algebra of $\mathfrak{A}$. Let $\mathscr{D}$ be a dense subspace of $\mathbb{S}$ such that $e \in \mathscr{D}$ and 
$\mathscr{H} \mathscr{D} \subset \mathscr{D}$ (for example, $\mathfrak{A}$ or the maximal unbounded Hilbert algebra $\left.L_{2}^{\omega}(\mathfrak{A})[5]\right) . \quad$ If $\delta$ is a quasi-bounded $*$-derivation of $\mathscr{C l}$ into $\mathscr{L}^{\sharp}(\mathscr{D},(\mathfrak{S})$ such that $\overline{\delta(A) \eta} \mathscr{C l}$ for each $A \in \mathscr{M}$, then it is spatial.

Proof. Since $\delta$ is quasi-bounded, there is an element $T$ of $\mathscr{L}^{\sharp}(\mathscr{D}, \mathbb{S})$ such that $T^{-1} \in \mathscr{B}(\mathbb{S})$ and $\delta\left(\mathscr{C}_{u}\right)$ is a bounded subset of the normed space $\mathfrak{M}_{T}^{*}$. It is easily showed that $\mathfrak{A} \subset \mathscr{D}$ and $S B^{\prime} \xi=$ $B^{\prime} S \xi$ for all $S \in \mathfrak{Q}_{\hat{\delta}}, B^{\prime} \in \mathscr{C l}^{\prime}$ and $\xi \in \mathfrak{A}$. This implies that $G_{\hat{\delta}, T}$ is noncontracting. In fact, for each pair of elements $S_{1} \neq S_{2}$ in $\mathfrak{D}_{\hat{o}}$ and $U \in \mathscr{C}_{u}$ we have

$$
\begin{aligned}
\left\|U^{*}\left(S_{1}-S_{2}\right) U e\right\| & =\left\|\left(S_{1}-S_{2}\right) \overline{\pi^{\prime}(u)} e\right\| \\
& =\left\|\overline{\pi^{\prime}(u)}\left(S_{1}-S_{2}\right) e\right\| \\
& =\left\|\left(S_{1}-S_{2}\right) e\right\| \\
& \neq 0,
\end{aligned}
$$

where $\pi\left(\operatorname{resp} . \pi^{\prime}\right)$ is the left (resp. right) regular representation of $\mathfrak{A}$ and $U=\overline{\pi(u)}$ for $u \in \mathfrak{A}$. Hence it follows from Proposition 4.8 that $\delta$ is spatial.

THEOREM 4.11. Let $\mathscr{C}$ be the left von Neumann algebra of a maximal Hilbert algebra $\mathfrak{A}$ with identity $e$, $\$ 5$ the completion of $\mathfrak{A}$ and let $\mathscr{D}$ be a countably dominated subspace of $\& 5$ by a sequence $\left\{T_{n}\right\}$ of closed operators such that $e \in \mathscr{D}$ and $\mathscr{M} \mathscr{D} \subset \mathscr{D}$. If $\delta$ is a $\left(t_{w} \rightarrow t_{q w}^{\mathscr{O}}\right)$-continuous (or $\left(t_{s} \rightarrow t_{s}^{\mathscr{O}}\right),\left(t_{o w} \rightarrow t_{q \sigma w}^{\mathscr{O}}\right),\left(t_{\sigma s} \rightarrow t_{\sigma s}^{\mathscr{O}}\right)$-continuous) *derivation in $\mathscr{L}$, then it can be extended to a spatial *-derivation $\hat{\delta}$ of $\mathscr{C l}$ into $\mathscr{L}^{\sharp}(\mathscr{D},(S)$.

Proof. This follows from Lemma 3.7 and Lemma 4.10 .

We next examine the spatiality of derivations of $\mathscr{L}$ into $\mathfrak{M}_{T}^{\sharp}$ when $\bar{T} \eta \mathscr{C l}^{\prime}$ (or $\bar{T} \eta \mathscr{C}$ ).

Suppose that $\delta$ is a derivation of $\mathscr{C}$ into $\mathfrak{M}_{T}^{*}$, where $T \in \mathscr{L}_{c}(\mathscr{D}$, (S) and $\overline{T^{-1}} \in \mathscr{B}(\mathbb{S})$. We set

$$
\delta_{T}(A)=\overline{\delta(A) T^{-1}} \text { for } A \in \mathscr{M} .
$$

It then follows from Lemma 2.1 that $\delta_{T}$ is a linear map of $\mathscr{C}$ into $\mathscr{B}(\mathbb{B})$, and so we have the following diagram:

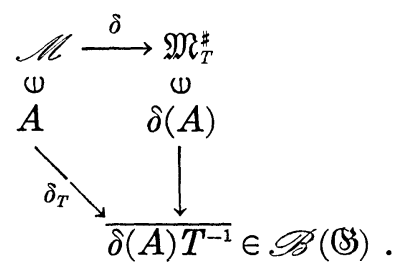


Furthermore, we have the following result, by a simple calculation

LEMmA 4.12. If $T \in \mathscr{L}_{c}\left(\mathscr{D},(S)\right.$ and $\overline{T^{-1}} \in \mathscr{C}^{\prime}$, then the linear map $\delta_{T}$ is a derivation of $\mathscr{C l}$ into $\mathscr{B}(\mathbb{S})$.

Definition 4.13. A von Neumann algebra $\mathscr{C}$ on $\mathbb{B S}$ is said to have the property (C) if every derivation $\delta$ of $\mathscr{C}$ into $\mathscr{B}(\mathbb{S})$ is inner; that is, $\delta$ is implemented by an element of $\mathscr{B}(\mathbb{S})$.

We note [3] that if $\mathscr{C}$ is of type I or properly infinite then has the property (C).

Proposition 4.14. Let $\mathscr{D}$ be a dense subspace in a Hilbert space (8) and let $\mathscr{C}$ be a von Neumann algebra on (S) with the property (C) and $\mathscr{H} \mathscr{D} \subset \mathscr{D}$. If $\delta$ is a *-derivation of $\mathscr{C l}$ into $\mathfrak{M}_{T}$ where $T \in \mathscr{L}_{c}(\mathscr{D}, \mathbb{S})$ and $T^{-1} \in \mathscr{C}^{\prime}$, then there exists an element $B_{0}$ of $\mathscr{B}(\mathbb{S})$ such that

$$
\delta(A) \xi=\left[B_{0} T, A\right] \xi
$$

for all $A \in \mathscr{M}$ and $\xi \in \mathscr{D}$, i.e., $\delta$ is spatial.

Proof. By Lemma $4.12, \delta_{T}$ is a derivation of $\mathscr{C l}$ into $\mathscr{B}(\mathbb{S})$. Hence it follows by the assumption that there exists an element $B_{0}$ of $\mathscr{B}(\mathbb{S})$ such that

$$
\delta_{T}(A)=\left[B_{0}, A\right] \text { for all } A \in \mathscr{C} .
$$

This implies that

$$
\delta(A) \xi=\left[B_{0} T, A\right] \xi \quad \text { for all } A \in \mathscr{C l} \text { and } \xi \in \mathscr{D} .
$$

THEOREM 4.15. Let $\mathscr{C l}$ be a von Neumann algebra on a Hilbert space $\mathbb{B}$ with the property (C) and let $\delta$ be a *-derivation in $\mathscr{M}$. Suppose that there exists a countably dominated subspace $\mathscr{D}$ of $\mathbb{B}$ by a sequence $\left\{T_{n}\right\}$ of closed operators $T_{n} \eta \mathscr{C l}^{\prime}$ such that $\delta$ is $\left(t_{w} \rightarrow t_{q w}^{\mathscr{O}}\right)$ continuous (or $\left(t_{s} \rightarrow t_{s}^{\mathscr{O}}\right),\left(t_{\sigma} \rightarrow t_{q \sigma w}^{\mathscr{O}}\right),\left(t_{\sigma s} \rightarrow t_{\sigma s}^{\mathscr{O}}\right)$-continuous). Then there

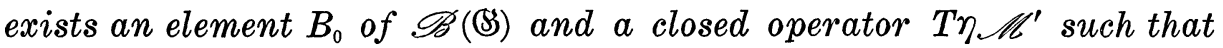

$$
\delta(A) \xi=\left[B_{0} T, A\right] \xi \quad \text { for all } A \in \mathscr{D}(\delta) \text { and } \xi \in \mathscr{D} .
$$

Proof. Since $T_{n} \eta \mathscr{C}^{\prime}$ for $n=1,2, \cdots$, we have $\mathscr{C} \mathscr{D} \subset \mathscr{D}$. It follows from Lemma 3.3 that $\delta$ is extended to a $\left(t_{w} \rightarrow t_{q w}^{\mathscr{S}}\right)$-continuous *-derivation $\hat{\delta}$ of $\mathscr{C}$ into $\mathscr{L}^{\sharp}(\mathscr{D}, \mathbb{S})$. Furthermore, by Lemma 2.6 $\hat{\delta}$ is quasi-bounded, i.e., $\hat{\delta}(\mathscr{C}) \subset \mathfrak{M}_{I+\left|T_{n}\right|}^{*}$ for some $n$. Hence the theorem follows from Proposition 4.14. 
COROLLARY 4.16. Let \& be the completion of a Hilbert algebra $\mathfrak{A}, \mathscr{C}$ the left von Neumann algebra of $\mathfrak{A}$ and let $J$ be the unitary involution on $\mathfrak{A}$. Suppose that $\mathscr{C}$ has the property (C) and there exists a countably dominated subspace $\mathscr{D}$ of \&S by a sequence $\left\{T_{n}\right\}$ of closed operators $T_{n} \eta \mathscr{C l}$ such that $J \mathscr{D}=\mathscr{D}$. If $\delta$ is $a\left(t_{w} \rightarrow t_{q w}^{\mathscr{O}}\right)$ continuous (or $\left(t_{s} \rightarrow t_{s}^{\mathscr{O}}\right),\left(t_{\sigma w} \rightarrow t_{q \sigma w}^{\mathscr{O}}\right),\left(t_{\text {os }} \rightarrow t_{\sigma s}^{\mathscr{O}}\right)$-continuous $) *$-derivation in $\mathscr{C}$, then it is extended to spatial derivation $\hat{\delta}$ of $\mathscr{C}$ into $\mathscr{L}^{\sharp}(\mathscr{D}$, (S).

Proof. We put

$$
T_{n}^{\prime}=J T_{n} J, \quad n=1,2, \cdots .
$$

It is then proved that $\mathscr{D}$ is countably dominated by the sequence $\left\{T_{n}^{\prime}\right\}$ of closed operators $T_{n}^{\prime} \eta \mathscr{C}^{\prime}$. Hence the corollary follows from Theorem 4.15 .

Proposition 4.17. Let th be a von Neumann algebra on a Hilbert space $\mathbb{B}$ and let $\delta$ be a *-derivation in $\mathscr{A}$. If there exists a countably dominated subspace $\mathscr{D}$ of \& by a sequence $\left\{T_{n}\right\}$ of closed operators $T_{n} \eta \mathscr{C} \cap \mathscr{L}^{\prime}$ such that $\delta$ is $\left(t_{w} \rightarrow t_{q w}^{\mathscr{O}}\right)$-continuous, then $\delta$ is extended to a spatial *-derivation $\hat{\delta}$ of $\mathscr{H}$ into $\mathscr{L}^{\sharp}(\mathscr{D},(\mathbb{S})$.

Proof. By Lemma 3.3 and Lemma 2.6, $\delta$ is extended to a quasibounded *-derivation $\hat{\delta}$ of $\mathscr{C}$ into $\mathfrak{M}_{T}^{\sharp}$, where $T \in \mathscr{L}^{\sharp}(\mathscr{D}, \mathbb{S})$ and $\overline{T^{-1}} \in \mathscr{L} \cap \mathscr{C}^{\prime}$, satisfying $\hat{\delta}\left(A^{*}\right)^{*} C \xi=C \hat{\delta}(A) \xi$ for each $A \in \mathscr{M}, C \in \mathscr{C}^{\prime}$ and $\xi \in \mathscr{D}$. Since $\mathscr{H} \mathscr{D} \subset \mathscr{D}$ and $\mathscr{C}^{\prime} \mathscr{D} \subset \mathscr{D}$, we have $\overline{\hat{\delta}(A)} \eta \mathscr{M}$ for each $A \in \mathscr{l}$. Since $T \in \mathscr{H} \cap \mathscr{H}^{\prime}, \hat{\delta}_{T}$ is a derivation of $\mathscr{C l}$ into $\mathscr{L}$. Hence, there exists an element $B_{0}$ of $\mathscr{C}$ such that

$$
\widehat{\delta}_{T}(A)=\left[B_{0}, A\right] \text { for each } A \in \mathscr{C},
$$

so that

$$
\hat{\delta}(A) \xi=\left[B_{0} T, A\right] \xi \text { for all } A \in \mathscr{C} \text { and } \xi \in \mathscr{D} .
$$

\section{REFERENCES}

1. D. Arnal and J. P. Jurzak, Topological aspects of algebras of unbounded operators.

J. Functional Analysis, 24 (1977), 397-425.

2. O. Bratteli, Unbounded derivations of operator algebras, CNRS Colloquium on Algebras of Operators and their Applications to Mathematical Physics, Marseille, 25-29 June, 1977.

3. E. Christensen, Extensions of derivations, J. Functional Analysis, 27 (1978), 234-247.

4. J. Dixmier, "Les algèbres d'opérateurs dans l'espace Hilbertien", $2^{\circ}$ édition, GauthierVillars, Paris, 1969. 
5. A. Inoue, On a class of unbounded operator algebras II, Pacific J. Math., 66 (1976), 411-431.

6. A. Inoue, K. Kuriyama and S. Ôta, Topologies on unbounded operator algebras, Mem. Fac. Sci. Kyushu-Univ., 33 (1979), 355-375.

7. A. Inoue and S. Ôta, Derivations on algebras of unbounded operators, Trans. Math. Soc., 261 (1980), 567-577.

8. G. Lassner, Topological algebras of operators, Rep. Math. Phys., 3 (1972), 279-293.

9. I. Namioka and E. Asplund, A geometric proof of Ryll-Nardzewski's fixed point theorem, Bull. Amer. Math. Soc., 73 (1967), 443-445.

10. S. Sakai, Derivations of $W^{*}$-algebras, Ann. Math., 83 (1966), 273-279.

11. —, $C^{*}$-algebras and $W^{*}$-algebras, Berlin-Heidelberg-New York, SpringerVerlag, 1971.

12. - Recent developments in the theory of unbounded derivations in $C^{*}$-algebras, Proceeding, Second Japan-USA Seminor, Los Angeles, April 18-22, 1977. (Lecture Notes in Math. 650, Springer-Verlag, Berlin, 1978).

13. K. Schmüdgen, Some remarks on topologization of unbounded operator algebras, preprint.

Received May 15, 1980.

FUKUOKa UNIVERSITY

FUKUOKA, JAPAN

KYUSHU UNIVERSITY

FUKUOKA, JAPAN

AND

Nitgata UNIVERSITY

NigGata, JAPAN 


\section{PACIFIC JOURNAL OF MATHEMATICS}

\section{EDITORS}

DoNALD BABBITT (Managing Editor)

University of California

Los Angeles, CA 90024

Hugo Rossi

University of Utah

Salt Lake City, UT 84112

C. C. MOORE and ANDREw OGG

University of California

Berkeley, CA 94720
J. DugundJI

Department of Mathematics

University of Southern California

Los Angeles, CA 90007

R. FinN and J. Milgram

Stanford University

Stanford, CA 94305

ASSOCIATE EDITORS
R. ARENS
E. F. BECKENBACH
B. H. NeumanN
F. WOLF
K. YoshidA

\section{SUPPORTING INSTITUTIONS}

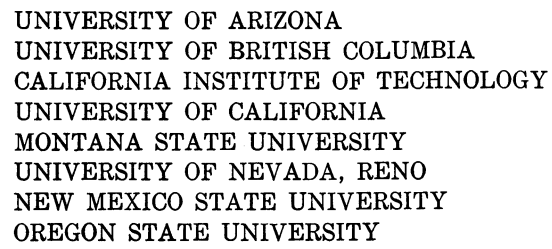

UNIVERSITY OF ARIZONA

UNIVERSITY OF BRITISH COLUMBIA

CALIFORNIA INSTITUTE OF TECHNOLOGY

UNIVERSITY OF CALIFORNIA

MONTANA STATE UNIVERSITY

UNIVERSITY OF NEVADA, RENO

NEW MEXICO STATE UNIVERSITY OREGON STATE UNIVERSITY

\author{
UNIVERSITY OF OREGON \\ UNIVERSITY OF SOUTHERN CALIFORNIA \\ STANFORD UNIVERSITY \\ UNIVERSITY OF HAWAII \\ UNIVERSITY OF TOKYO \\ UNIVERSITY OF UTAH \\ WASHINGTON STATE UNIVERSITY \\ UNIVERSITY OF WASHINGTON
}

The Supporting Institutions listed above contribute to the cost of publication of this Journal, but they are not owners or publishers and have no responsibility for its content or policies.

Mathematical papers intended for publication in the Pacific Journal of Mathematics should be in typed form or offset-reproduced, (not dittoed), double spaced with large margins. Please do not use built up fractions in the text of the manuscript. However, you may use them in the displayed equations. Underline Greek letters in red, German in green, and script in blue. The first paragraph or two must be capable of being used separately as a synopsis of the entire paper. Please propose a heading for the odd numbered pages of less than 35 characters. Manuscripts, in triplicate, may be sent to any one of the editors. Please classify according to the scheme of Math. Reviews, Index to Vol. 39. Supply name and address of author to whom proofs should be sent. All other communications should be addressed to the managing editor, or Elaine Barth, University of California, Los Angeles, California, 90024.

50 reprints to each author are provided free for each article, only if page charges have been substantially paid. Additional copies may be obtained at cost in multiples of 50 .

The Pacific Journal of Mathematics is issued monthly as of January 1966. Regular subscription rate: $\$ 102.00$ a year (6 Vols., 12 issues). Special rate: $\$ 51.00$ a year to individual members of supporting institutions.

Subscriptions, orders for numbers issued in the last three calendar years, and changes of address shoud be sent to Pacific Journal of Mathematics, P.O. Box 969, Carmel Valley, CA 93924, U.S.A. Old back numbers obtainable from Kraus Periodicals Co., Route 100, Millwood, NY 10546.

\footnotetext{
PUBLISHED BY PACIFIC JOURNAL OF MATHEMATICS, A NON-PROFIT CORPORATION

Printed at Kokusai Bunken Insatsusha (International Academic Printing Co., Ltd.). 8-8, 3-chome, Takadanobaba, Shinjuku-ku, Tokyo 160, Japan.
} 


\section{Pacific Journal of Mathematics}

\section{Vol. 96, No. 2 December, 1981}

Gerald A. Beer, A natural topology for upper semicontinuous functions and

a Baire category dual for convergence in measure $\ldots \ldots \ldots \ldots \ldots \ldots 251$

Georgia Benkart and J. Marshall Osborn, An investigation of real

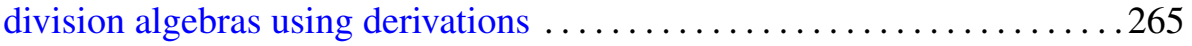

Donald Ian Cartwright and John R. McMullen, A structural criterion for the existence of infinite Sidon sets ........................ 301

Philip Hanlon, The fixed-point partition lattices $\ldots \ldots \ldots \ldots \ldots \ldots \ldots \ldots$

Eric Hayashi, The spectral density of a strongly mixing stationary Gaussian process

Chung-Wu Ho and Charles E. Morris, Jr., A graph-theoretic proof of

Sharkovsky's theorem on the periodic points of continuous functions . ...361

Sara Hurvitz, The automorphism groups of spaces and fibrations ....... 371

Atsushi Inoue, Schoichi Ota and Jun Tomiyama, Derivations of operator algebras into spaces of unbounded operators . .................. 389

Wolfgang B. Jurkat and Gary Sampson, On weak restricted estimates and endpoint problems for convolutions with oscillating kernels. I ........ 405

Georgios Koumoullis, Some topological properties of spaces of

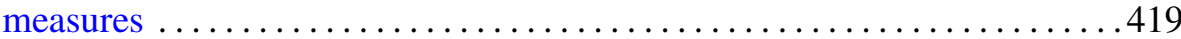

Wen Hsiung Lin, Algebraic Kahn-Priddy theorem ................. 435

Michael John McAsey, Invariant subspaces of nonselfadjoint crossed

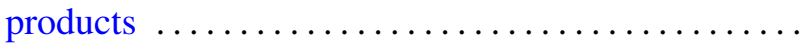

Justin Peters, Entropy of automorphisms on L.C.A. groups

Saburou Saitoh, A characterization of the adjoint $L$-kernel of Szegó type 